University of South Florida

DIGITAL COMMONS

Digital Commons @ University of

@ UNIVERSITY OF SOUTH FLORIDA

South Florida

$1-1-2017$

\title{
2017 Work Plan USF Sarasota-Manatee
}

USF

Follow this and additional works at: https://digitalcommons.usf.edu/usf_accountability_reports

\section{Scholar Commons Citation}

USF, "2017 Work Plan USF Sarasota-Manatee" (2017). USF Accountability Reports. 65.

https://digitalcommons.usf.edu/usf_accountability_reports/65

This Article is brought to you for free and open access by the USF Archives at Digital Commons @ University of South Florida. It has been accepted for inclusion in USF Accountability Reports by an authorized administrator of Digital Commons @ University of South Florida. For more information, please contact digitalcommons@usf.edu. 


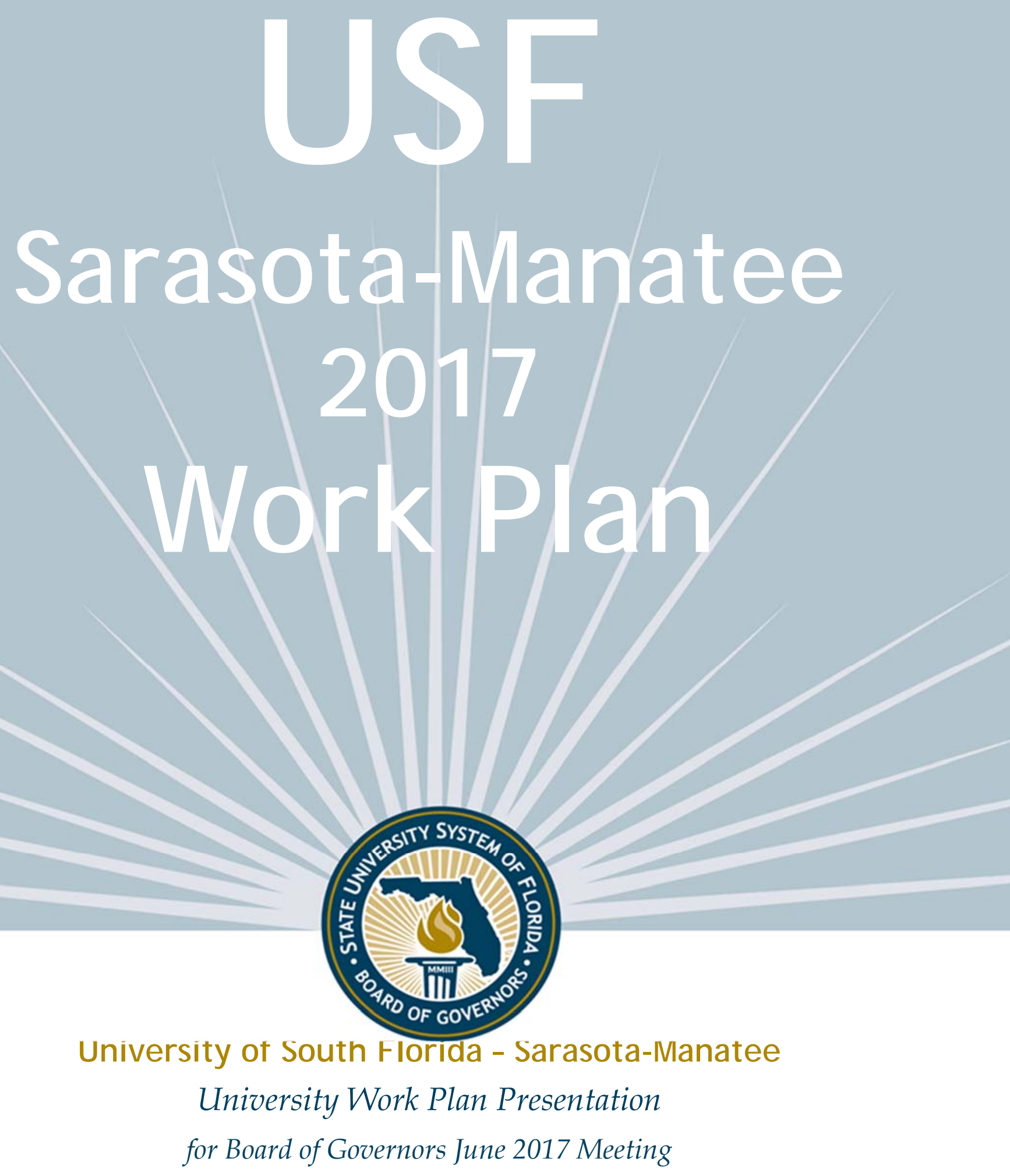

STATE UNIVERSITY SYSTEM of FLORIDA $\mid$ Board of Governors 


\section{INTRODUCTION}

The State University System of Florida has developed three tools that aid in guiding the System's future.

1) The Board of Governors' 2025 System Strategic Plan is driven by prospective goals and associated metrics that set future benchmarks for the System;

2) The Board's Annual Accountability Report provides retrospective tracking with year-over-year and longer time periods for how the System is progressing toward its goals;

3) Institutional Work Plans connect the two and create an opportunity for greater dialogue relative to how each institution contributes to the System's overall vision.

These three documents assist the Board with strategic planning and with setting short-, mid- and long-term goals. They al so enhance the System's commitment to accountability and driving improvements in three primary areas of focus: 1) academic quality, 2) operational efficiency, and 3) return on investment.

The Board will use these documents to help advocate for all System institutions and foster even greater coordination with the institutions and their Boards of Trustees.

Once a Work Plan is approved by each institution's respective Boards of Trustees, the Board of Governors will review and consider the plan for potential acceptance of the one-year metric goals. Longer-term components will inform future agendas of the Board's Strategic Planning Committee. The Board's acceptance of a work plan does not constitute approval of any particular component, nor does it supersede any necessary approval processes that may be required for each component. 


\section{TABLE OF CONTENTS}

1. STRATEGY
a. Mission Statement
b. Vision Statement
c. Statement of Strategy
d. Strengths and Opportunities
e. Key Initiatives \& Investments

2. PERFORMANCE BASED FUNDING METRICS

\section{PREEMINENT RESEARCH UNIVERSITY METRICS}

4. KEY PERFORMANCE INDICATORS
a. Teaching \& Learning
b. Scholarship, Research and Innovation
c. Institution Specific Goals

\section{ENROLLMENT PLANNING}

\section{ACADEMIC PROGRAM COORDINATION}

\section{UNIVERSITY REVENUES}

\section{TUITION, FEES AND HOUSING PROJECTIONS}

\section{DEFINITIONS}




\section{MSSION STATEMENT (What is your purpose?)}

The University of South Florida Sarasota-Manatee provides high quality bachelor's and graduate-level education and scholarly activity in a personalized learning community that prepares successful leaders and responsible citizens.

\section{VSION STATEMENT (What do you aspire to?)}

As a valued member of the USF System, the University of South Florida Sarasota-Manatee will be nationally recognized as a student-centered, research-focused, community-engaged university with significant economic and cultural impact.

\section{STATEMENT OF STRATEGY (How will you get there?)}

Given your mission, vision, strengths and available resources, provide a brief description of your market and your strategy for addressing and leading it.

USF Sarasota-Manatee's primary focus is to provide quality, affordable, bachelor's and master's degrees that address the specific higher education needs. USF Sarasota-Manatee serves its market by offering a mix of classroom and online instruction at times that are convenient to a commuter student population. USF Sarasota-Manatee partners with local businesses, non-profits, and educational institutions to meet the need for quality internship opportunities for students and producing talented graduates for today's global workforce. 


\section{STRENGTHS AND OPPORTUNITIES (within 3 years)}

What are your core capabilities, opportunities and challenges for improvement?

USF Sarasota-Manatee provides access to a personalized, learning experience that permits students to "learn where they earn." The institution capitalizes on its low student-to-faculty ratio by offering individualized attention from high quality and committed faculty to ensure that students are supported, retained, and successful. As a member of the USF System, USF Sarasota-Manatee benefits from the efficiency of shared resources, a unified brand that yields identity and impact, and the opportunity for collaboration with other USF System institutions - including via tailored 2+2 programs to meet the needs of the Tampa Bay region without unnecessary duplication of programs.

One such example of this is a burgeoning new partnership between USF and USF Sarasota-Manatee in engineering. USF Sarasota-Manatee students complete two years of pre-engineering course work, earning an associate in arts certificate, and then transfer to the USF campus in Tampa to obtain a baccalaureate in engineering. This allows the USF System to serve the growing workforce need for engineers without building another costly engineering program across the Bay. USF Sarasota-Manatee continues to seek similar partnerships across the USF System and with other community partners to increase its profile and serve its mission as a regional institution dedicated to supporting the knowledge economy.

\section{KEY INITIATIVES \& INMESTMENTS (within 3 years)}

Describe your top three key initiatives for the next three years that will drive improvement in Academic Quality, Operational Efficiency, and Return on Investment.

\section{Reach the goals of the USF Sarasota-Manatee 2015-20 Strategic Plan, Focus on Quality 2020:}

USF Sarasota-Manatee faculty, staff, students, alumni, governance boards, and community representatives selected seven major goals for the Strategic Plan, which aligns with the USF System's guiding principles and SUS goals: (1) Support Student Success, (2) Enhance Campus Life, (3) Engage in Intentional Enrollment Management, (4) Strengthen Teaching \& Learning, (5) Promote Scholarly Activity, (6) Maximize Community Engagement, and (7) Cultivate Resources. A broad array of strategies are being implemented to meet the goals of the Strategic Plan and Performance-Based Funding. Of the seven PBF metrics reported in USFSM's 2015-16 Annual Accountability Report, USFSM met or significantly improved on five and has developed further strategies to improve the other two.

\section{Expand STEM and Areas of Strategic Emphasis offerings:}

USF Sarasota-Manatee's College of Science and Mathematics opened in Fall 2016 with a founding dean, who is deepening the commitment to applied science and mathematics offerings. USF SarasotaManatee is pursuing the following key initiatives: (1) expansion of laboratory and research space, (2) enrollment growth in STEM and Areas of Strategic Emphasis, (3) initiation of an Accounting scholarship to attract highly qualified students, and (4) a partnership with the College of Nursing at USF in Tampa to offer seamless admission of biology graduates into a second bachelor's degree in Nursing. 


\section{Preserve, grow, and diversify USF Sarasota-Manatee resources:}

USF Sarasota-Manatee will strategically increase efforts in grant-writing, private fundraising, and public-private partnership development to maximize its resources in order to invest in those areas that will help it advance its Strategic Plan goals, performance-based funding metrics, and other USF System initiatives. 


\section{PERFORMANCE BASED FUNDING METRICS (ACTUAL | GOALS)}

1. Percent of Bachelor's Graduates Enrolled or Employed $(\$ 25,000+)$ [within one-year, anywhere in the Nation]

\begin{tabular}{ccccc|cccc}
$2010-11$ & $2011-12$ & $2012-13$ & $2013-14$ & $2014-15$ & $2015-16$ & $2016-17$ & $2017-18$ & $2018-19$ \\
\hline. &. & $65.5 \%$ & $62.8 \%$ & $69.6 \%$ & $72.8 \%$ & $73.8 \%$ & $74.8 \%$ & $75.8 \%$
\end{tabular}

2. Median Wages of Bachelor's Graduates Employed Full-time [within one-year, anywhere in the Nation]

\begin{tabular}{ccccc|cccc}
$2010-11$ & $2011-12$ & $2012-13$ & $2013-14$ & $2014-15$ & $2015-16$ & $2016-17$ & $2017-18$ & $2018-19$ \\
\hline$\$ 33,600$ & $\$ 33,200$ & $\$ 36,000$ & $\$ 36,200$ & $\$ 39,000$ & $\$ 40,700$ & $\$ 41,200$ & $\$ 41,700$ & $\$ 42,200$
\end{tabular}

3. Average Cost to the Student [Net Tuition \& Fees per 120 Credit Hours for Resident Undergraduates]

\begin{tabular}{lllll|llll}
$2011-12$ & $2012-13$ & $2013-14$ & $2014-15$ & $2015-16$ & $2016-17$ & $2017-18$ & $2018-19$ & $2019-20$ \\
\hline
\end{tabular}

Reported at the USF System Level

4. FTIC Six-Year Graduation Rate

\begin{tabular}{ccccc|cccc}
$2006-12$ & $2007-13$ & $2008-14$ & $2009-15$ & $2010-16$ & $2011-17$ & $2012-18$ & $2013-19$ & $2014-20$ \\
\hline$\cdot$ & $\cdot$ & $\cdot$ & $\cdot$ & $\cdot$ & $\cdot$ & $\cdot$ & $55.0 \%$ & $60.0 \%$
\end{tabular}

5. Academic Progress Rate [Second Year Retention Rate with At Least a 2.0 GPA]

\begin{tabular}{ccccc|cccc}
$2011-12$ & $2012-13$ & $2013-14$ & $2014-15$ & $2015-16$ & $2016-17$ & $2017-18$ & $2018-19$ & $2019-20$ \\
\hline$\cdot$ & $\cdot$ & $69.9 \%$ & $78.7 \%$ & $74.7 \%$ & $82.0 \%$ & $84.0 \%$ & $86.0 \%$ & $90.0 \%$
\end{tabular}

6. Percentage of Bachelor's Degrees Awarded within Programs of Strategic Emphasis

\begin{tabular}{llllllllll}
$2011-12$ & $2012-13$ & $2013-14$ & $2014-15$ & $2015-16$ & $2016-17$ & $2017-18$ & $2018-19$ & $2019-20$ \\
\hline $34.4 \%$ & $39.8 \%$ & $33.9 \%$ & $41.1 \%$ & $37.0 \%$ & $42.0 \%$ & $45.0 \%$ & $48.0 \%$ & $50.0 \%$
\end{tabular}

7. University Access Rate [Percent of Undergraduates with a Pell grant]

\begin{tabular}{ccccc|cccc} 
FALL 2011 & FALL 2012 & FALL 2013 & FALL 2014 & FALL 2015 & FALL 2016 & FALL 2017 & FALL 2018 & FALL 2019 \\
\hline $49.9 \%$ & $41.7 \%$ & $40.7 \%$ & $45.2 \%$ & $44.4 \%$ & $44.4 \%$ & $44.4 \%$ & $45.0 \%$ & $45.0 \%$
\end{tabular}

8. Percentage of Graduate Degrees Awarded within Programs of Strategic Emphasis

\begin{tabular}{lllll|llll}
$\mathbf{2} 011-12$ & $\mathbf{2 0 1 2}-13$ & $\mathbf{2 0 1 3 - 1 4}$ & $\mathbf{2 0 1 4 - 1 5}$ & $\mathbf{2 0 1 5 - 1 6}$ & $\mathbf{2 0 1 6 - 1 7}$ & $\mathbf{2 0 1 7 - 1 8}$ & $\mathbf{2 0 1 8 - 1 9}$ & $\mathbf{2 0 1 9 - 2 0}$ \\
\hline $43.1 \%$ & $30.0 \%$ & $27.3 \%$ & $12.0 \%$ & $24.5 \%$ & $12.0 \%$ & $20.0 \%$ & $25.0 \%$ & $30.0 \%$
\end{tabular}

9. BOG Choice: Percent of Baccalaureate Degrees Awarded Without Excess Hours

\begin{tabular}{lllll|llll}
$2011-12$ & $2012-13$ & $2013-14$ & $2014-15$ & $2015-16$ & $2016-17$ & $2017-18$ & $2018-19$ & $2019-20$ \\
\hline $60.0 \%$ & $71.3 \%$ & $75.4 \%$ & $71.7 \%$ & $75.7 \%$ & $78.0 \%$ & $80.0 \%$ & $81.0 \%$ & $82.0 \%$
\end{tabular}

10. BOT Choice: Postdoctoral Appointees

\begin{tabular}{ccccc|cccc} 
Fall 2010 & Fall 2011 & Fall 2012 & Fall 2013 & Fall 2014 & Fall 2015 & Fall 2016 & Fall 2017 & Fall 2018 \\
\hline 0 & 0 & 0 & 0 & 0 & 5 & 5 & 5 & 5 \\
\hline
\end{tabular}

Note: Metrics are defined in appendix. For more information about the PBF model visit: http://www.flbog.edu/about/budget/performance funding.php. 


\section{KEY PERFORMANCE INDICATORS (ACTUAL | GOALS)}

Teaching \& Learning Metrics (from the 2025 System Strategic Plan that are not included in the PBF section)

1. Public University National Ranking [based on BOG's official list of publications]

\begin{tabular}{ccccc|cccc}
2013 & 2014 & 2015 & 2016 & 2017 & 2018 & 2019 & 2020 & 2021 \\
\hline$\cdot$ & $\cdot$ & $\cdot$ & $\cdot$ & $\cdot$ & & $\cdot$ & $\cdot$ & $\cdot$
\end{tabular}

\begin{tabular}{ccccc|cccc} 
Fall 2012 & Fall 2013 & Fall 2014 & Fall 2015 & Fall 2016 & Fall 2017 & Fall 2018 & Fall 2019 & Fall 2020 \\
\hline & $12 \%$ & $30 \%$ & $26 \%$ & $29 \%$ & $30 \%$ & $31 \%$ & $32 \%$ & $33 \%$
\end{tabular}

3. Professional Licensure \& Certification Exam Pass Rates Above Benchmarks

\begin{tabular}{ccccc|cccc}
$\mathbf{2 0 1 1 - 1 2}$ & $2012-13$ & $2013-14$ & $2014-15$ & $2015-16$ & $2016-17$ & $2017-18$ & $2018-19$ & $2019-20$ \\
\hline & $\cdot$ & $\cdot$ & $\cdot$ & $\cdot$ & $\cdot$ & $\cdot$ & $\cdot$ & $\cdot$ \\
4. Time & $\cdot$ & $\cdot$ & & & & & & \\
$2011-12$ & $2012-13$ & $2013-14$ & $2014-15$ & $2015-16$ & $2016-17$ & $2017-18$ & $2018-19$ & $2019-20$ \\
\hline$\cdot$ & $\cdot$ & $\cdot$ & $\cdot$ & $\cdot$ & 4.2 & 4.2 & 4.2 & 4.2
\end{tabular}

5. Four-Year FTIC Graduation Rates [full-time students only]

2008-12 2009-13 2010-14 2011-15 2012-16

\begin{tabular}{cccc}
$2013-17$ & $2014-18$ & $2015-19$ & $2016-20$ \\
\hline $35 \%$ & $36 \%$ & $38 \%$ & $40 \%$
\end{tabular}

6. Bachelor's Degrees Awarded [First Majors Only]

\begin{tabular}{ccccc|cccc}
$2011-12$ & $2012-13$ & $2013-14$ & $2014-15$ & $2015-16$ & $2016-17$ & $2017-18$ & $2018-19$ & $2019-20$ \\
\hline 514 & 556 & 490 & 474 & 464 & 442 & 457 & 473 & 497
\end{tabular}

7. Graduate Degrees Awarded [First Majors Only]

\begin{tabular}{ccccc|cccc}
$2011-12$ & $2012-13$ & $2013-14$ & $2014-15$ & $2015-16$ & $2016-17$ & $2017-18$ & $2018-19$ & $2019-20$ \\
\hline 72 & 60 & 55 & 50 & 53 & 73 & 76 & 78 & 82
\end{tabular}

8. Bachelor's Degrees Awarded to African-American \& Hispanic Students

\begin{tabular}{ccccc|cccc}
$2011-12$ & $2012-13$ & $2013-14$ & $2014-15$ & $2015-16$ & $2016-17$ & $2017-18$ & $2018-19$ & $2019-20$ \\
\hline $17 \%$ & $18 \%$ & $15 \%$ & $24 \%$ & $21 \%$ & $21 \%$ & $22 \%$ & $23 \%$ & $24 \%$
\end{tabular}

9. Percentage of Adult (Aged 25+) Undergraduates Enrolled

\begin{tabular}{ccccc|cccc} 
Fall 2012 & Fall 2013 & Fall 2014 & Fall 2015 & Fall 2016 & Fall 2017 & Fall 2018 & Fall 2019 & Fall 2020 \\
\hline $59 \%$ & $55 \%$ & $51 \%$ & $48 \%$ & $44 \%$ & $43 \%$ & $43 \%$ & $42 \%$ & $42 \%$
\end{tabular}

10. Percent of Undergraduate FTE in Online Courses

\begin{tabular}{ccccc|cccc}
$2011-12$ & $2012-13$ & $2013-14$ & $2014-15$ & $2015-16$ & $2016-17$ & $2017-18$ & $2018-19$ & $2019-20$ \\
\hline $45 \%$ & $49 \%$ & $50 \%$ & $49 \%$ & $52 \%$ & $53 \%$ & $55 \%$ & $55 \%$ & $55 \%$
\end{tabular}

11. Percent of Bachelor's Degrees in STEM \& Health

\begin{tabular}{ccccc|cccc}
$2011-12$ & $2012-13$ & $2013-14$ & $2014-15$ & $2015-16$ & $2016-17$ & $2017-18$ & $2018-19$ & $2019-20$ \\
\hline $5 \%$ & $17 \%$ & $14 \%$ & $19 \%$ & $15 \%$ & $19 \%$ & $22 \%$ & $25 \%$ & $28 \%$
\end{tabular}

12. Percent of Graduate Degrees in STEM \& Health

\begin{tabular}{ccccc|cccc}
$2011-12$ & $2012-13$ & $2013-14$ & $2014-15$ & $2015-16$ & $2016-17$ & $2017-18$ & $2018-19$ & $2019-20$ \\
\hline $0.0 \%$ & $0.0 \%$ & $0.0 \%$ & $0.0 \%$ & $0.0 \%$ & $0.0 \%$ & $0.0 \%$ & $0.0 \%$ & $0.0 \%$ \\
\hline
\end{tabular}




\section{Institution Specific Goals (optional)}

To further distinguish the university's distinctive mission, the university may choose to provide additional metric goals that are based on the university's own strategic plan.

\begin{tabular}{lccccccccc}
\hline & Fall & Fall & Fall & Fall & Fall & Fall & Fall & Fall & Fall \\
& 2012 & 2013 & 2014 & 2015 & 2016 & 2017 & 2018 & 2019 & 2020 \\
\hline $\begin{array}{l}\text { \% of Non-White Faculty } \& \\
\text { Staff }\end{array}$ & $14 \%$ & $14 \%$ & $18 \%$ & $19 \%$ & $20 \%$ & $20 \%$ & $20 \%$ & $21 \%$ & $21 \%$ \\
\hline & Fall & Fall & Fall & Fall & Fall & Fall & Fall & Fall & Fall \\
& 2012 & 2013 & 2014 & 2015 & 2016 & 2017 & 2018 & 2019 & 2020 \\
\hline $\begin{array}{l}\text { \% of Hispanic \& African } \\
\text { American Students }\end{array}$ & $19 \%$ & $19 \%$ & $20 \%$ & $20 \%$ & $19 \%$ & $22 \%$ & $23 \%$ & $24 \%$ & $25 \%$ \\
\hline
\end{tabular}




\section{ENROLLMENT PLANNING (ACTUAL | PLAN)}

Planned Headcount Enrollment by Student Type (for all students at all campuses)

\begin{tabular}{|c|c|c|c|c|c|c|c|c|c|}
\hline & $\begin{array}{c}\text { FALL } 2012 \\
\text { ACTUAL }\end{array}$ & $\begin{array}{c}\text { FALL } 2013 \\
\text { ACTUAL }\end{array}$ & $\begin{array}{c}\text { FALL } 2014 \\
\text { ACTUAL }\end{array}$ & $\begin{array}{c}\text { FALL } 2015 \\
\text { ACTUAL }\end{array}$ & $\begin{array}{c}\text { FALL } 2016 \\
\text { ACTUAL }\end{array}$ & $\begin{array}{c}\text { FALL } 2017 \\
\text { PLAN }\end{array}$ & $\begin{array}{c}\text { FALL } 2018 \\
\text { PLAN }\end{array}$ & $\begin{array}{c}\text { FALL } 2019 \\
\text { PLAN }\end{array}$ & $\begin{array}{c}\text { FALL } 2020 \\
\text { PLAN }\end{array}$ \\
\hline \multicolumn{10}{|l|}{ UNDERGRADUATE } \\
\hline FTIC (Regular Admit) & 30 & 109 & 195 & 268 & 315 & 335 & 352 & 372 & 394 \\
\hline FTIC (Profile Admit) & 0 & 0 & 1 & 3 & 6 & 3 & 3 & 3 & 4 \\
\hline FCS AA Transfers & 918 & 826 & 789 & 781 & 737 & 770 & 809 & 853 & 904 \\
\hline Other AA Transfers & 181 & 169 & 155 & 159 & 159 & 166 & 174 & 184 & 195 \\
\hline Post-Baccalaureates & 0 & 0 & 152 & 127 & 137 & 143 & 150 & 159 & 168 \\
\hline Other Undergraduates & 583 & 567 & 400 & 419 & 440 & 460 & 483 & 509 & 540 \\
\hline Subtotal & 1,712 & 1,671 & 1,692 & 1,757 & 1,794 & 1,878 & 1,972 & 2,080 & 2,205 \\
\hline \multicolumn{10}{|l|}{ GRADUATE } \\
\hline Master's & 140 & 128 & 120 & 174 & 178 & 186 & 195 & 206 & 218 \\
\hline Research Doctoral & & & - & $\cdot$ & . & $\cdot$ & - & . & . \\
\hline Professional Doctoral & & & . & . & 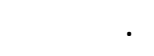 & 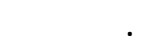 & . & . & . \\
\hline Subtotal & 140 & 128 & 120 & 174 & 178 & 186 & 195 & 206 & 218 \\
\hline \multicolumn{10}{|l|}{ UNCLASSIFIED } \\
\hline H.S. Dual Enrolled & & & & & & & & & \\
\hline Other ${ }^{1}$ & 100 & 88 & 105 & 113 & 108 & 114 & 120 & 126 & 134 \\
\hline Subtotal & 100 & 88 & 105 & 113 & 108 & 114 & 120 & 126 & 134 \\
\hline TOTAL & 1,952 & 1,887 & 1,917 & 2,044 & 2,080 & 2,178 & 2,287 & 2,412 & 2,557 \\
\hline
\end{tabular}

Notes: This table reports the number of students enrolled at the university by student type categories. The student type for undergraduates is based on the Type of Student at Time of Most Recent Admission. The student type for graduates is based on the degree that is sought and the student CIP code. Unclassified refers to a student who has not yet been formally admitted into a degree program but is enrolled. The methodology for this table was revised at the June 2016 Data Administrator Workshop and matches the 2015-16 Accountability Report (Table 3A). The change improves how post-baccalaureate undergraduate students are counted. (1) 'Other Unclassified' students include Post-Baccalaureates who are not seeking a degree.

\section{Planned FTE Enrollment by Method of Instruction (for all students at all campuses)}

\begin{tabular}{|c|c|c|c|c|c|c|c|c|c|}
\hline & $\begin{array}{l}2011-12 \\
\text { ACTUAL }\end{array}$ & $\begin{array}{l}2012-13 \\
\text { ACTUAL }\end{array}$ & $\begin{array}{l}2013-14 \\
\text { ACTUAL }\end{array}$ & $\begin{array}{l}2014-15 \\
\text { ACTUAL }\end{array}$ & $\begin{array}{l}2015-16 \\
\text { ACTUAL }\end{array}$ & $\begin{array}{c}2016-17 \\
\text { PLAN }\end{array}$ & $\begin{array}{c}2017-18 \\
\text { PLAN }\end{array}$ & $\begin{array}{c}2018-19 \\
\text { PLAN }\end{array}$ & $\begin{array}{c}2019-20 \\
\text { PLAN }\end{array}$ \\
\hline \multicolumn{10}{|l|}{ UNDERGRADUATE } \\
\hline Distance (80-100\%) & 669 & 726 & 721 & 743 & 878 & 919 & 1,003 & 1,068 & 1,143 \\
\hline Hybrid (50-79\%) & 46 & 46 & 62 & 33 & 8 & 8 & 18 & 19 & 21 \\
\hline Classroom (0-50\%) & 764 & 709 & 663 & 743 & 787 & 818 & 803 & 854 & 914 \\
\hline Subtotal & 1,479 & 1,481 & 1,446 & 1,519 & 1,673 & 1,745 & 1,824 & 1,942 & 2,078 \\
\hline \multicolumn{10}{|l|}{ GRADUATE } \\
\hline Distance (80-100\%) & 29 & 30 & 31 & 45 & 51 & 54 & 59 & 63 & 68 \\
\hline Hybrid (50-79\%) & 5 & 5 & 6 & 6 & 2 & 2 & 5 & 6 & 6 \\
\hline Classroom (0-50\%) & 118 & 98 & 97 & 78 & 110 & 113 & 98 & 104 & 112 \\
\hline Subtotal & 152 & 133 & 134 & 129 & 163 & 169 & 163 & 173 & 186 \\
\hline
\end{tabular}

Note: Full-time Equivalent (FTE) student is a measure of instructional activity that is based on the number of credit hours that students enroll. FTE is based on the standard national definition, which divides undergraduate credit hours by 30 and graduate credit hours by 24 . Distance Learning is a course in which at least 80 percent of the direct instruction of the course is delivered using some form of technology when the student and instructor are separated by time or space, or both (per $1009.24(17)$, F.S.). Hybrid is a course where $50 \%$ to $79 \%$ of the instruction is delivered using some form of technology, when the student and instructor are separated by time or space, or both (per SUDS data element 2052). Classroom/Traditional, is a course in which less than $50 \%$ of the direct instruction of the course is delivered using some form of technology when the student and instructor are separated by time, space or both. This designation can include activities that do not occur in a classroom (ie, labs, internships, practica, clinicals, labs, etc) - see SUDS data element 2052. 


\section{ENROLLMENT PLANNING (continued)}

\section{Planned FTE Enrollment Plan by Student Level}

\begin{tabular}{|c|c|c|c|c|c|c|c|c|c|}
\hline & $\begin{array}{l}2015-16 \\
\text { ACTUAL }\end{array}$ & $\begin{array}{c}2016-17 \\
\text { ESTIMATE }\end{array}$ & $\begin{array}{c}2017-18 \\
\text { PLAN }\end{array}$ & $\begin{array}{l}\text { 2018-19 } \\
\text { PLAN }\end{array}$ & $\begin{array}{c}2019-20 \\
\text { PLAN }\end{array}$ & $\begin{array}{c}2020-21 \\
\text { PLAN }\end{array}$ & $\begin{array}{l}2021-22 \\
\text { PLAN }\end{array}$ & $\begin{array}{c}2022-23 \\
\text { PLAN }\end{array}$ & $\begin{array}{c}\text { Annual } \\
\text { Growth } \\
\text { Rate }^{*}\end{array}$ \\
\hline \multicolumn{10}{|c|}{ STATE FUNDABLE } \\
\hline \multicolumn{10}{|l|}{ RESIDENT } \\
\hline LOWER & 393 & 455 & 482 & 514 & 550 & 591 & 638 & 692 & $7.5 \%$ \\
\hline UPPER & 1,161 & 1,146 & 1,211 & 1,289 & 1,379 & 1,483 & 1,602 & 1,738 & $7.5 \%$ \\
\hline GRAD I & 145 & 141 & 144 & 154 & 164 & 177 & 191 & 207 & $7.5 \%$ \\
\hline GRAD ॥ & 0 & 0 & 0 & 0 & 0 & 0 & 0 & 0 & \\
\hline TOTAL & 1,699 & 1,742 & 1,837 & 1,956 & 2,093 & 2,250 & 2,430 & 2,637 & $7.5 \%$ \\
\hline \multicolumn{10}{|c|}{ NON RESIDENT } \\
\hline LOWER & 31 & 34 & 36 & 38 & 41 & 44 & 47 & 51 & $7.5 \%$ \\
\hline UPPER & 54 & 57 & 60 & 64 & 69 & 74 & 80 & 87 & $7.5 \%$ \\
\hline GRAD I & 15 & 13 & 13 & 14 & 15 & 16 & 18 & 19 & $7.5 \%$ \\
\hline GRAD ॥ & 0 & 0 & 0 & 0 & 0 & 0 & 0 & 0 & \\
\hline TOTAL & 100 & 104 & 110 & 117 & 125 & 134 & 145 & 157 & $7.5 \%$ \\
\hline \multicolumn{10}{|l|}{ TOTAL } \\
\hline LOWER & 424 & 489 & 518 & 552 & 590 & 635 & 686 & 744 & $7.5 \%$ \\
\hline UPPER & 1,215 & 1,203 & 1,271 & 1,353 & 1,448 & 1,557 & 1,681 & 1,824 & $7.5 \%$ \\
\hline GRAD I & 160 & 154 & 158 & 168 & 180 & 193 & 208 & 226 & $7.5 \%$ \\
\hline GRAD ॥ & 0 & 0 & 0 & 0 & 0 & 0 & 0 & 0 & \\
\hline TOTAL & 1,799 & 1,846 & 1,946 & 2,073 & 2,218 & 2,384 & 2,575 & 2,794 & $7.5 \%$ \\
\hline \multicolumn{10}{|c|}{ NOT STATE FUNDABLE } \\
\hline LOWER & 6 & 10 & 11 & 12 & 13 & 14 & 15 & 16 & $7.5 \%$ \\
\hline UPPER & 27 & 19 & 24 & 25 & 27 & 29 & 31 & 34 & $7.5 \%$ \\
\hline GRAD I & 2 & 0 & 5 & 6 & 6 & 6 & 7 & 8 & $7.5 \%$ \\
\hline GRAD ॥ & 0 & 0 & 0 & 0 & 0 & 0 & 0 & 0 & $7.5 \%$ \\
\hline TOTAL & 35 & 29 & 40 & 43 & 46 & 49 & 53 & 58 & $7.5 \%$ \\
\hline
\end{tabular}

Note: Full-time Equivalent (FTE) student is a measure of instructional activity that is based on the number of credit hours that students enroll. FTE is based on the standard national definition, which divides undergraduate credit hours by 30 and graduate credit hours by 24. Pursuant to section 1013.31, Florida Statutes, this data is used as a key factor in the calculation of facility space needs for university educational plant surveys. Note*: The Planned Annual Growth Rate is a compounded rate based on the following formula: (2022-23 value divided by the 2017-18 value) to the (1/5) exponent minus one. 


\section{ACADEMC PROGRAM COORDINATION}

\section{New Programs For Consideration by University in AY 2017-18}

The S.U.S. Council of Academic Vice Presidents (CAVP) Academic Program Coordination Work Group will review these programs as part of their on-going coordination efforts. The programs listed below are based on the 2016 Work Plan list for programs under consideration for 2017-18.

\begin{tabular}{|c|c|c|c|c|c|c|}
\hline PROGRAM TITLES & $\begin{array}{l}\text { CIP CODE } \\
\text { 6-digit }\end{array}$ & $\begin{array}{l}\text { AREA OF } \\
\text { STRATEGIC } \\
\text { EMPHASIS }\end{array}$ & $\begin{array}{l}\text { OTHER } \\
\text { UNIVERSITIES } \\
\text { WITH SAME } \\
\text { PROGRAM } \\
\end{array}$ & $\begin{array}{l}\text { OFFERED VIA } \\
\text { DISTANCE } \\
\text { LEARNING } \\
\text { IN SYSTEM } \\
\end{array}$ & $\begin{array}{l}\text { PROJECTED } \\
\text { ENROLLMENT } \\
\text { in 5th year }\end{array}$ & $\begin{array}{l}\text { PROPOSED } \\
\text { DATE OF } \\
\text { SUBMISSION } \\
\text { TO UBOT } \\
\end{array}$ \\
\hline \multicolumn{7}{|l|}{ BACHELOR'S PROGRAMS } \\
\hline $\begin{array}{l}\text { BS Risk Management/Insurance } \\
\text { (USFSM) }\end{array}$ & 52.1701 & $\begin{array}{c}\text { GAP } \\
\text { ANALYSIS }\end{array}$ & FSU & $0 \%$ & 25 & Spring 2018 \\
\hline
\end{tabular}

\section{MASTER'S, SPECIALIST AND OTHER ADVANCED MASTER'S PROGRAMS}

N/A

\section{DOCTORAL PROGRAMS}

N/A

New Programs For Consideration by University in 2018-19

These programs will be used in the 2017 Work Plan list for programs under consideration for 2018-20.

\begin{tabular}{|c|c|c|c|c|c|c|}
\hline PROGRAM TITLES & $\begin{array}{l}\text { CIP CODE } \\
\text { 6-digit }\end{array}$ & $\begin{array}{c}\text { AREA OF } \\
\text { STRATEGIC } \\
\text { EMPHASIS }\end{array}$ & $\begin{array}{l}\text { OTHER } \\
\text { UNIVERSITIES } \\
\text { WITH SAME } \\
\text { PROGRAM }\end{array}$ & $\begin{array}{l}\text { OFFERED VIA } \\
\text { DISTANCE } \\
\text { LEARNING } \\
\text { IN SYSTEM }\end{array}$ & $\begin{array}{l}\text { PROJECTED } \\
\text { ENROLLMENT } \\
\text { in 5th year }\end{array}$ & $\begin{array}{l}\text { PROPOSED } \\
\text { DATE OF } \\
\text { SUBMISSION } \\
\text { TO UBOT }\end{array}$ \\
\hline LOR'S PROGRAMS & & & & & & \\
\hline $\begin{array}{l}\text { Management Science } \\
\text { (USFSM) }\end{array}$ & 52.1301 & STEM & UF & $0 \%$ & 25 & Spring 2019 \\
\hline
\end{tabular}

\section{MASTER'S, SPECIALIST AND OTHER ADVANCED MASTER'S PROGRAMS}

Biology/Biological Sciences,

General (USFSM)
FAMU, FAU,

26.0101 STEM FIU, FSU, UCF,

UNF, UWF
$0 \%$

25

Spring 2019

\section{DOCTORAL PROGRAMS}

N/A 


\section{UNIVERSTYY REVENUES}

University Revenues (in Millions of Dollars)

EDUCATION \& GENERAL

\section{Main Operations}

State Funds

Tuition

SUBTOTAL
2015-16

Actual

$\$ 15.80$

$\$ 9.10$

$\$ 24.90$
2016-17

Estimates

\section{EDUCATION \& GENERAL TOTAL REVENUES}

$\$ 24.90$

$\$ 17.24$

$\$ 9.59$

$\$ 26.83$

$\$ 26.83$

Note: State funds include General Revenue funds, Lottery funds, Federal Stimulus funds, and Phosphate Research funds (for Polytechnic) appropriated by the Florida Legislature (as reported in the Annual Accountability Report). Actual tuition includes base tuition and tuition differential fee revenues for resident and non-resident undergraduate and graduate students net of waivers (as reported in the Annual Accountability Report).

\section{OTHER BUDGET ENTITIES}

\begin{tabular}{lcc}
\hline Auxiliary Enterprises & $\$ 2.60$ & $\$ 1.77$ \\
Contracts \& Grants & $\$ 0.20$ & $\$ 0.27$ \\
Local Funds & $\$ 1.30$ & $\$ 1.28$ \\
Faculty Practice Plans & $\$ 0$ & $\$ 0$ \\
\hline OTHER BUDGET ENTITIES TOTAL REVENUES & $\$ 4.1$ & $\$ 3.32$
\end{tabular}




\section{UNIVERSITY TUITION, FEES AND HOUSING PROJ ECTIONS}

University: USF Sarasota-Manatee

\begin{tabular}{|c|c|c|c|c|c|c|c|}
\hline \multirow{2}{*}{ Undergraduate Students } & \multicolumn{3}{|c|}{ 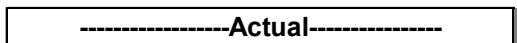 } & \multicolumn{4}{|c|}{ 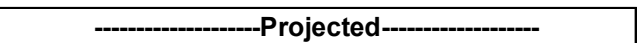 } \\
\hline & 2014-15 & 2015-16 & 2016-17 & 2017-18 & 2018-19 & 2019-20 & $2020-21$ \\
\hline \multicolumn{8}{|l|}{ Tuition: } \\
\hline Base Tuition - (0\% inc. for $2016-17$ to $2019-20)$ & $\$ 105.07$ & $\$ 105.07$ & $\$ 105.07$ & $\$ 105.07$ & $\$ 105.07$ & $\$ 105.07$ & $\$ 105.07$ \\
\hline Total Base Tuition \& Differential per Credit Hour & $\$ 140.21$ & $\$ 140.21$ & $\$ 140.21$ & $\$ 140.21$ & $\$ 140.21$ & $\$ 140.21$ & $\$ 140.21$ \\
\hline$\%$ Change & & $0.0 \%$ & $0.0 \%$ & $0.0 \%$ & $0.0 \%$ & $0.0 \%$ & $0.0 \%$ \\
\hline Capital Improvement $^{2}$ & $\$ 20.19$ & $\$ 20.19$ & $\$ 20.19$ & $\$ 20.19$ & $\$ 20.19$ & $\$ 20.19$ & $\$ 20.19$ \\
\hline Activity \& Service & $\$ 4.03$ & $\$ 4.03$ & $\$ 4.03$ & $\$ 4.03$ & $\$ 4.03$ & $\$ 4.03$ & $\$ 4.03$ \\
\hline Health & $\$ 4.23$ & $\$ 4.23$ & $\$ 4.23$ & $\$ 4.23$ & $\$ 4.23$ & $\$ 4.23$ & $\$ 4.23$ \\
\hline \multicolumn{8}{|l|}{ Athletic } \\
\hline Transportation Access & $\$ 5.25$ & $\$ 5.25$ & $\$ 5.25$ & $\$ 5.25$ & $\$ 5.25$ & $\$ 5.25$ & $\$ 5.25$ \\
\hline \multicolumn{8}{|l|}{$\begin{array}{l}\text { Technology } \\
{ }^{1} \\
\text { Green Fee (USF, NCF, UWF only) } \\
\text { Student Life \& Services Fee (UNF only) } \\
\text { Marshall Center Fee (USF only) } \\
\text { Student Affairs Facility Use Fee (FSU only) }\end{array}$} \\
\hline Total Fees & $\$ 45.71$ & $\$ 45.71$ & $\$ 45.71$ & $\$ 45.71$ & $\$ 45.71$ & $\$ 45.71$ & $\$ 45.71$ \\
\hline \multirow{2}{*}{\begin{tabular}{|c|} 
Total Tuition and Fees per Credit Hour \\
$\%$ Change \\
\end{tabular}} & $\$ 185.92$ & $\$ 185.92$ & $\$ 185.92$ & $\$ 185.92$ & $\$ 185.92$ & $\$ 185.92$ & $\$ 185.92$ \\
\hline & & $0.0 \%$ & $0.0 \%$ & $0.0 \%$ & $0.0 \%$ & $0.0 \%$ & $0.0 \%$ \\
\hline \multicolumn{8}{|l|}{$\begin{array}{l}\text { Activity \& Service } \\
\text { Health }\end{array}$} \\
\hline Athletic & $\$ 5.00$ & $\$ 5.00$ & $\$ 5.00$ & $\$ 5.00$ & $\$ 5.00$ & $\$ 5.00$ & $\$ 5.00$ \\
\hline \multicolumn{8}{|l|}{ Transportation Access } \\
\hline \multicolumn{8}{|l|}{ Marshall Center Fee (USF only) } \\
\hline \multicolumn{8}{|l|}{ Student Affairs Facility Use Fee (FSU only) } \\
\hline \multicolumn{8}{|l|}{ List any new fee proposed } \\
\hline \multicolumn{8}{|l|}{ Out-of-State Fees } \\
\hline Out-of-State Undergraduate Fee & $\$ 346.50$ & $\$ 346.50$ & $\$ 346.50$ & $\$ 346.50$ & $\$ 346.50$ & $\$ 346.50$ & $\$ 346.50$ \\
\hline Out-of-State Undergraduate Student Financial $\mathrm{Aid}^{3}$ & $\$ 17.32$ & $\$ 17.32$ & $\$ 17.32$ & $\$ 17.32$ & $\$ 17.32$ & $\$ 17.32$ & $\$ 17.32$ \\
\hline \multirow{2}{*}{\begin{tabular}{|c|} 
Total per credit hour \\
$\%$ Change
\end{tabular}} & $\$ 363.82$ & $\$ 363.82$ & $\$ 363.82$ & $\$ 363.82$ & $\$ 363.82$ & $\$ 363.82$ & $\$ 363.82$ \\
\hline & & $0.0 \%$ & $0.0 \%$ & $0.0 \%$ & $0.0 \%$ & $0.0 \%$ & $0.0 \%$ \\
\hline Total Tuition for 30 Credit Hours & $\$ 14,601.30$ & $\$ 14,601.30$ & $\$ 14,601.30$ & $\$ 14,601.30$ & $\$ 14,601.30$ & $\$ 14,601.30$ & $\$ 14,601.30$ \\
\hline \multirow{2}{*}{$\begin{array}{l}\text { Total Fees for } 30 \text { Credit Hours } \\
\text { Total Tuition and Fees for } 30 \text { Credit Hours }\end{array}$} & $\$ 1,900.90$ & $\$ 1,900.90$ & $\$ 1,900.90$ & $\$ 1,900.90$ & $\$ 1,900.90$ & $\$ 1,900.90$ & $\$ 1,900.90$ \\
\hline & $\$ 16,502.20$ & $\$ 16,502.20$ & $\$ 16,502.20$ & $\$ 16,502.20$ & $\$ 16,502.20$ & $\$ 16,502.20$ & $\$ 16,502.20$ \\
\hline \multirow{2}{*}{\begin{tabular}{|c} 
\$Change \\
$\%$ Change \\
\end{tabular}} & & $\$ 0.00$ & $\$ 0.00$ & $\$ 0.00$ & $\$ 0.00$ & $\$ 0.00$ & $\$ 0.00$ \\
\hline & & $0.0 \%$ & $0.0 \%$ & $0.0 \%$ & $0.0 \%$ & $0.0 \%$ & $0.0 \%$ \\
\hline \multicolumn{8}{|l|}{ Housing/Dining ${ }^{4}$} \\
\hline \$ Change & & N/A & N/A & N/A & N/A & N/A & N/A \\
\hline$\%$ Change & & N/A & N/A & N/A & N/A & N/A & N/A \\
\hline${ }^{1}$ can be no more than $5 \%$ of tuition. & & tutti & d the out-of-s & fee. & & & \\
\hline${ }^{2}$ as approved by the Board of Governors. & 4 combine the $\mathrm{mc}$ & ost popular housin & g and dining plan & ovided to students & & & \\
\hline & cenort curre & tion differentia & al. Only UF or & an reflest $\mathrm{no}$ & . & & \\
\hline
\end{tabular}




\section{DEFINITIONS}

\section{Performance Based Funding}

\section{Percent of Bachelor's Graduates Enrolled or Employed $(\$ 25,000+)$} One Year After Graduation

\section{Cost to the Student} Net Tuition \& Fees for Resident Undergraduates per 120 Credit Hours

\section{Median Wages of Bachelor's Graduates Employed Full-time One Year After Graduation}

This metric is based on the percentage of a graduating class of bachelor's degree recipients who are enrolled or employed (earning at least $\$ 25,000$ ) somewhere in the United States. Students who do not have valid social security numbers and are not found enrolled are excluded. This data now includes non-Florida data from 41 states and districts, including the District of Columbia and Puerto Rico. Sources: Accountability Report (Table 40). State University Database System (SUDS), Florida Education \& Training Placement Information Program (FETPIP) analysis of Wage Record Interchange System (WRIS2) and Federal Employment Data Exchange (FEDES), and National Student Clearinghouse (NSC).

This metric is based on annualized Unemployment Insurance (UI) wage data from the fourth fiscal quarter after graduation for bachelor's recipients. This data does not include individuals who are self-employed, employed by the military, those without a valid social security number, or making less than minimum wage. This data now includes non-Florida data from 41 states and districts, including the District of Columbia and Puerto Rico. Sources: Accountability Report (Table 40). State University Database System (SUDS), Florida Education \& Training Placement Information Program (FETPIP) analysis of Wage Record Interchange System (WRIS2) and Federal Employment Data Exchange (FEDES), and National Student Clearinghouse (NSC).

This metric is based on resident undergraduate student tuition and fees, books and supplies as calculated by the College Board (which serves as a proxy until a university work group makes an alternative recommendation), the average number of credit hours attempted by students who were admitted as FTIC and graduated with a bachelor's degree for programs that requires 120 credit hours, and financial aid (grants, scholarships and waivers) provided to resident undergraduate students (does not include unclassified students). Source: Accountability Report (Table 1D) - which, combines the Legislature's annual General Appropriations Act, university required fees and several files (HTD, SFA, SIF) within SUDS.

\section{Six Year FTIC Graduation Rate}

This metric is based on the percentage of first-time-in-college (FTIC) students who started in the Fall (or summer continuing to Fall) term and had graduated from the same institution within six years. Source: Accountability Report (Table 4D).
This metric is based on the percentage of first-time-in-college (FTIC) students who started in the Fall (or summer continuing to Fall) term and were enrolled full-time in their first semester and were still enrolled in the same institution during the Fall term following their first year with had a grade point average (GPA) of at least 2.0 at the end of their first year (Fall, Spring, Summer). Source: Accountability Report (Table 4B).

\section{University Access Rate Percent of Undergraduates with a Pell-grant}

7. Bachelor's Degrees within
Programs of Strategic
Emphasis

This metric is based the number of undergraduates, enrolled during the fall term, who received a Pell-grant during the fall term. Unclassified students, who are not eligible for Pellgrants, were excluded from this metric. Source: Accountability Report (Table 3E).

This metric is based on the number of baccalaureate degrees awarded within the programs designated by the Board of Governors as 'Programs of Strategic Emphasis'. A student who has multiple majors in the subset of targeted Classification of Instruction Program codes will be counted twice (i.e., double-majors are included). Source: Accountability Report (Table $4 \mathrm{H}$ ). 


\section{8a. Graduate Degrees within Programs of Strategic Emphasis}

\author{
8b. Freshmen in Top 10\% \\ of High School Class \\ Applies to: NCF
}

This metric is based on the number of graduate degrees awarded within the programs designated by the Board of Governors as 'Programs of Strategic Emphasis'. A student who has multiple majors in the subset of targeted Classification of Instruction Program codes will be counted twice (i.e., double-majors are included). Source: Accountability Report (Table $5 C)$.

Percent of all degree-seeking, first-time, first-year (freshman) students who had high school class rank within the top $10 \%$ of their graduating high school class.

Source: New College of Florida as reported to the Common Data Set (C10).

BOG Choice Metrics

\section{9a. Percent of Bachelor's Degrees Without Excess Hours}

This metric is based on the percentage of baccalaureate degrees awarded within $110 \%$ of the credit hours required for a degree based on the Board of Governors Academic Program Inventory.

Note: It is important to note that the statutory provisions of the "Excess Hour Surcharge" $(1009.286$, FS) have been modified several times by the Florida Legislature, resulting in a phased-in approach that has created three different cohorts of students with different requirements. The performance funding metric data is based on the latest statutory requirements that mandates $110 \%$ of required hours as the threshold. In accordance with statute, this metric excludes the following types of student credits (ie, accelerated mechanisms, remedial coursework, non-native credit hours that are not used toward the degree, non-native credit hours from failed, incomplete, withdrawn, or repeated courses, credit hours from internship programs, credit hours up to 10 foreign language credit hours, and credit hours earned in military science courses that are part of the Reserve Officers' Training Corps (ROTC) program). Source: State University Database System (SUDS). humanities, science, engineering and health fields as reported in the annual 'Top American Research Universities' report. Twenty-three of the most prominent awards are considered, including: Getty Scholars in Residence, Guggenheim Fellows, Howard Hughes Medical Institute Investigators, MacArthur Foundation Fellows, National Endowment for the Humanities (NEH) Fellows, National Medal of Science and National Medal of Technology, Robert Wood Johnson Policy Fellows, Sloan Research Fellows, Woodrow Wilson Fellows, to name a few awards. Source: Center for Measuring University Performance, Annual Report of the Top American Research Universities (TARU).

This metric is based on the number of Top 50 university rankings that NCF earned from the following list of publications: Princeton Review, Fiske Guide, QS World University Ranking, Times Higher Education World University Ranking, Academic Ranking of World University, US News and World Report National University, US News and World Report National Public University, US News and World Report Liberal Arts Colleges, Forbes, Kiplinger, Washington Monthly Liberal Arts Colleges, Washington Monthly National University, and Center for Measuring University Performance. Source: Board of Governors staff review.

\section{BOT Choice Metrics}

10a. Percent of R\&D Expenditures Funded from External Sources FAMU

10b. Bachelor's Degrees Awarded to Minorities FAU, FGCU, FIU
This metric reports the amount of research expenditures that was funded from federal, private industry and other (non-state and non-institutional) sources.

Source: National Science Foundation annual survey of Higher Education Research and Development (HERD).

This metric is the number, or percentage, of baccalaureate degrees granted in an academic year to Non-Hispanic Black and Hispanic students. This metric does not include students classified as Non-Resident Alien or students with a missing race code.

Source: State University Database System (SUDS). 
10c. National Rank Higher than Predicted by the Financial Resources Ranking Based on U.S. and World News FSU
This metric is based on the difference between the Financial Resources rank and the overall University rank. U.S. News measures financial resources by using a two-year average spending per student on instruction, research, student services and related educational expenditures - spending on sports, dorms and hospitals doesn't count.

Source: US News and World Report's annual National University rankings.

\section{0d. Percent of Undergraduate Seniors Participating in a Research Course NCF \\ 10e. Number of Bachelor Degrees Awarded Annually UCF}

This metric is based on the percentage of undergraduate seniors who participate in a research course during their senior year.

Source: New College of Florida.

This metric is the number of baccalaureate degrees granted in an academic year. Students who earned two distinct degrees in the same academic year were counted twice; students who completed multiple majors or tracks were only counted once.

Source: State University Database System (SUDS).

\section{0f. Number of Licenses/Options Executed Annually UF}

10g. Percent of Undergraduate FTE in Online Courses UNF

Number of Postdoctoral
Appointees
USF

Percentage of Adult Undergraduates Enrolled UWF
This metric is the total number of licenses and options executed annually as reported to Association of Technology Managers (AUTM). The benchmarks are based on UF's rank within AAU institutions. Source: Accountability Report (Table 6A), University of Florida.

This metric is based on the percentage of undergraduate full-time equivalent (FTE) students enrolled in online courses. The FTE student is a measure of instructional activity that is based on the number of credit hours that students enroll by course level. Distance Learning is a course in which at least 80 percent of the direct instruction of the course is delivered using some form of technology when the student and instructor are separated by time or space, or both (per 1009.24(17), F.S.). Source: Accountability Report (Table 3C), State University Database System (SUDS).

This metric is based on the number of post-doctoral appointees at the beginning of the academic year. A postdoctoral researcher has recently earned a doctoral (or foreign equivalent) degree and has a temporary paid appointment to focus on specialized research/scholarship under the supervision of a senior scholar.

Source: National Science Foundation/National Institutes of Health annual Survey of Graduate Students and Postdoctorates in Science and Engineering (GSS).

This metric is based on the percentage of undergraduates (enrolled during the fall term) who are at least 25 years old at the time of enrollment. This includes undergraduates who are not degree-seeking, or unclassified.

Source: State University Database System (SUDS).

\section{Preeminent Research University Funding Metrics}

Average GPA and SAT Score

Public University Nationa Ranking
An average weighted grade point average of 4.0 or higher and an average SAT score of 1200 or higher for fall semester incoming freshmen, as reported annually in the admissions data that universities submit to the Board of Governors. This data includes registered FTIC (student type='B','E') with an admission action of admitted or provisionally admitted ('A', 'P', 'X').

A top-50 ranking on at least two well-known and highly respected national public university rankings, reflecting national preeminence, using most recent rankings, includes: Princeton Review, Fiske Guide, QS World University Ranking, Times Higher Education World University Ranking, Academic Ranking of World University, US News and World Report National University, US News and World Report National Public University, US News and World Report Liberal Arts Colleges, Forbes, Kiplinger, Washington Monthly Liberal Arts Colleges, Washington Monthly National University, and Center for Measuring University Performance. 
Freshman Retention Rate (Full-time, FTIC)

6-year Graduation Rate (Full-time, FTIC)
Freshman Retention Rate (Full-time, FTIC) as reported annually to the Integrated Postsecondary Education Data System (IPEDS). The retention rates that are reported in the Board's annual Accountability report are preliminary because they are based on student enrollment in their second fall term as reported by the 28th calendar day following the first day of class. When the Board of Governors reports final retention rates to IPEDS in the Spring (usually the first week of April), that data is based on the student enrollment data as reported after the Fall semester has been completed. The preliminary and final retention rates are nearly identical when rounded to the nearest whole number.

Cohorts are based on undergraduate students who enter the institution in the Fall term (or Summer term and continue into the Fall term). Percent Graduated is based on federal rate and does not include students who originally enroll as part-time students, or who transfer into the institution. This metric complies with the requirements of the federal Student Right to Know Act that requires institutions to report the completion status at $150 \%$ of normal time (or six years). For more information about how this data is calculated, see: http://www.flbog.edu/about/budget/docs/performance funding/PBF GRADUATION and RETENTIO N Methodology FINAL.pdf.

National Academy Memberships held by faculty as reported by the Center for Measuring University Performance in the Top American Research Universities (TARU) annual report or the official membership directories maintained by each national academy.

Science \& Engineering Research Expenditures, including federal research expenditures as reported annually to the National Science Foundation (NSF).
Science \& Engineering Research Expenditures (\$M)
Total S\&E research expenditures in non-medical sciences as reported to the NSF. This removes medical sciences funds ( $9 F$ \& $12 F$ in HERD survey) from the total S\&E amount.
Non-Medical

Science \& Engineering

Research Expenditures (\$M)

National Ranking in S.T.E.M. Research Expenditures

Patents Awarded

(3 calendar years)
The NSF identifies 8 broad disciplines within Science \& Engineering (Computer Science, Engineering, Environmental Science, Life Science, Mathematical Sciences, Physical Sciences, Psychology, Social Sciences). The rankings by discipline are determined by BOG staff using the NSF WebCaspar database.

Total patents awarded by the United States Patent and Trademark Office (USPTO) for the most recent three calendar year period. Due to a year-lag in published reports, Board of Governors staff query the USPTO database with a query that only counts utility patents:"(AN/"University Name" AND ISD/yyyymmdd->yyyymmdd AND APT/1)".
Doctoral Degrees Awarded Annually

Number of Post-Doctoral Appointees

Endowment Size (\$M)
Doctoral degrees awarded annually, as reported annually in the Board of Governors Accountability Report.

The number of Postdoctoral Appointees awarded annually, as reported in the TARU annual report. This data is based on National Science Foundation/National Institutes of Health annual Survey of Graduate Students and Postdoctorates in Science and Engineering (GSS).

This data comes from the National Association of College and University Business Officers (NACUBO) and Commonfund Institute's annual report of Market Value of Endowment Assets - which, due to timing, may release the next fiscal year's data after the Board of Governors Accountability report is published. 


\section{Key Performance Indicators \\ Teaching \& Learning Metrics}

Freshmen in Top $10 \%$

of HS Graduating Class

Professional/Licensure

Exam First-time Pass Rates

Average Time to Degree

for FTIC in 120hr programs

FTIC Graduation Rates

In 4 years (or less)

Bachelor's Degrees Awarded

Graduate Degrees Awarded

Bachelor's Degrees Awarded To African-American and Hispanic Students

Adult (Aged 25+)

Undergraduates Enrolled

Fall term

Percent of Undergraduate FTE Enrolled in Online Courses

Percent of Bachelor's Degrees in STEM \& Health

Percent of Graduate Degrees in STEM \& Health
Percent of all degree-seeking, first-time, first-year (freshman) students who had high school class rank within the top $10 \%$ of their graduating high school class. As reported by the university to the Common Data Set (C10).

The number of exams with first-time pass rates above and below the national or state average, as reported in the annual Accountability report, including: Nursing, Law, Medicine (3 subtests), Veterinary, Pharmacy, Dental (2 subtests), Physical Therapy, and Occupational Therapy.

This metric is the number of years between the start date (using date of most recent admission) and the end date (using the last month in the term degree was granted) for a graduating class of first-time, single-major baccalaureates in 120 credit hour programs within a (Summer, Fall, Spring) year.

As reported in the annual Accountability report (table 4D), First-time-in-college (FTIC) cohort is defined as undergraduates entering in fall term (or summer continuing to fall) with fewer than 12 hours earned since high school graduation. The rate is the percentage of the initial cohort that has either graduated from or is still enrolled in the same institution by the fourth academic year. Both full-time and part-time students are used in the calculation. The initial cohort is revised to remove students, who have allowable exclusions as defined by IPEDS, from the cohort.

This is a count of baccalaureate degrees awarded as reported in the annual Accountability Report (Table 4G).

This is a count of graduate degrees awarded as reported in the Accountability Report (Table $5 B)$.

Non-Hispanic Black and Hispanic do not include students classified as Non-Resident Alien or students with a missing race code - as reported in the Accountability Report (table 4I).

Students who earn two distinct degrees in the same term are counted twice - whether their degrees are from the same six-digit CIP code or different CIP codes. Students who earn only one degree are counted once - even if they completed multiple majors or tracks. Percentage of Degrees is based on the number of baccalaureate degrees awarded to non-Hispanic Black and Hispanic students divided by the total degrees awarded - excluding those awarded to non-resident aliens and unreported.

This metric is based on the age of the student at the time of enrollment (not upon entry). Age acts as a surrogate variable that captures a large, heterogeneous population of adult students who often have family and work responsibilities as well as other life circumstances that can interfere with successful completion of educational objectives.

Full-time Equivalent (FTE) student is a measure of instructional activity that is based on the number of credit hours that students enroll. FTE is based on the US definition, which divides undergraduate credit hours by 30. Distance Learning is a course in which at least 80 percent of the direct instruction of the course is delivered using some form of technology when the student and instructor are separated by time or space, or both (per 1009.24(17), F.S.). The percentage of baccalaureate degrees that are classified as STEM by the Board of Governors in the SUS program inventory as reported in the annual Accountability Report (Table 4H).

The percentage of baccalaureate degrees that are classified as STEM by the Board of Governors in the SUS program inventory as reported in the annual Accountability Report (Table 5C). 


\section{Key Performance Indicators (continued) \\ Scholarship, Research \& Innovation Metrics}

Awards include: American Council of Learned Societies (ACLS) Fellows, Beckman Young Investigators, Burroughs Wellcome Fund Career Awards, Cottrell Scholars, Fulbright American Scholars, Getty Scholars in Residence, Guggenheim Fellows, Howard Hughes Medical Institute Investigators, Lasker Medical Research Awards, MacArthur Foundation Fellows, Andrew W. Mellon Foundation Distinguished Achievement Awards, National

Faculty Awards Endowment for the Humanities (NEH) Fellows, National Humanities Center Fellows, National Institutes of Health (NIH) MERIT, National Medal of Science and National Medal of Technology, NSF CAREER awards (excluding those who are also PECASE winners), Newberry Library Long-term Fellows, Pew Scholars in Biomedicine, Presidential Early Career Awards for Scientists and Engineers (PECASE), Robert Wood Johnson Policy Fellows, Searle Scholars, Sloan Research Fellows, Woodrow Wilson Fellows. As reported by the Top American Research Universities - see: http://mup.asu.edu/research data.html.

Total Research Expenditures $(\$ M)$

Percent of R\&D Expenditures funded from External Sources

Licenses/Options Executed
Total expenditures for all research activities (including non-science and engineering activities) as reported in the National Science Foundation annual survey of Higher Education Research and Development (HERD).

This metric reports the amount of research expenditures that was funded from federal, private industry and other (non-state and non-institutional) sources.

Source: National Science Foundation annual survey of Higher Education Research and Development (HERD).

Licenses/options executed in the fiscal year for all technologies as reported in the annual Accountability Report (table 6A).

Number of Start-up Companies

The number of start-up companies that were dependent upon the licensing of University technology for initiation as reported in the annual Accountability Report (table 6A). 